\title{
Risk of lymph node metastases from intramucosal gastric cancer in relation to histological types: how to manage the mixed histological type for endoscopic submucosal dissection
}

\author{
Kohei Takizawa $\cdot$ Hiroyuki Ono $\cdot$ Naomi Kakushima $\cdot$ Masaki Tanaka \\ Noriaki Hasuike · Hiroyuki Matsubayashi • Yuichiro Yamagichi • \\ Etsuro Bando $\cdot$ Masanori Terashima $\cdot$ Kimihide Kusafuka $\cdot$ Takashi Nakajima
}

Received: 7 August 2012/ Accepted: 10 November 2012/Published online: 29 November 2012

(c) The International Gastric Cancer Association and The Japanese Gastric Cancer Association 2012

\begin{abstract}
Background The behavior of early gastric cancer (EGC) with mixed-type histology (differentiated and undifferentiated) is incompletely understood. This study aimed to clarify the clinicopathological features of EGC with mixedtype histology in relation to lymph node (LN) metastasis. Methods Clinicopathological data from 410 patients who underwent surgical resection for intramucosal EGC were reviewed. Lesions were classified into four types according to the proportion of differentiated and undifferentiated components at histopathology: pure differentiated (PD) type, mixed predominantly differentiated (MD) type, mixed predominantly undifferentiated (MU) type, and pure undifferentiated (PU) type. We examined the clinicopathological differences between PD and MD, and between PU and $\mathrm{MU}$, and the rate of LN metastasis according to tumor size and ulceration.
\end{abstract}

Meeting presentations: 8th International Gastric Cancer Congress 2009.

K. Takizawa $(\bowtie) \cdot$ H. Ono $\cdot$ N. Kakushima $\cdot$ M. Tanaka

H. Matsubayashi · Y. Yamagichi

Endoscopy Division, Shizuoka Cancer Center,

1007 Shimonagakubo Nagaizumi-cho, Sunto-gun,

Shizuoka 411-8777, Japan

e-mail: k.takizawa@scchr.jp

N. Hasuike

Gastroenterology Division, Sano Hospital, Hyogo, Japan

E. Bando $\cdot$ M. Terashima

Gastric Surgery Division, Shizuoka Cancer Center,

Nagaizumi-cho, Shizuoka, Japan

K. Kusafuka · T. Nakajima

Pathology Division, Shizuoka Cancer Center,

Nagaizumi-cho, Shizuoka, Japan
Results Moderately differentiated adenocarcinoma was the primary component in MD relative to PD (90.7 vs. $46.1 \%)$. Signet ring cell carcinoma was the main component in PU relative to MU (81.5 vs. $33.3 \%)$. LN metastasis was more common in MU than PU (19.0 vs. $6.0 \%)$. For intramucosal tumors larger than $20 \mathrm{~mm}$ without lymphovascular invasion and without ulceration, the rate of LN metastasis was $0 \%$ for MD and $24 \%$ for MU. For intramucosal lesions less than $30 \mathrm{~mm}$ with ulceration but without lymphovascular invasion, the rate of LN metastasis was $0 \%$ for MD and $20 \%$ for MU.

Conclusions Histologically mixed-type EGC with a predominantly undifferentiated component should be managed as an undifferentiated-type tumor. Further investigation is required to determine whether mixed-type EGC with a predominantly differentiated component could be managed the same way as a differentiated-type EGC.

Keywords Gastric neoplasms - Differentiated type · Undifferentiated type $\cdot$ Mixed type

\section{Introduction}

Endoscopic submucosal dissection (ESD) allows en-bloc resection of large superficial lesions and is a widely applied treatment for early gastric cancer (EGC) with little risk of lymph node (LN) metastasis [1-3]. Pathologically, gastric cancer can be broadly divided into two types according to the presence or absence of tubular structures: differentiated and undifferentiated types [4]. Characteristically, undifferentiated gastric cancer carries a higher risk of LN metastasis than differentiated gastric cancer [5-9]. However, some tumors contain a mixture of differentiated and undifferentiated components. 
Precise histopathological evaluation of EGC is now possible because of en-bloc resection using ESD; this has introduced dilemmas when a mixture of histological types is identified in the same lesion. According to the Japanese Classification of Gastric Carcinoma [4], gastric cancers showing a mixture of differentiated and undifferentiated components are classified according to the predominant histological type. To determine indications for ESD, many studies have examined surgically resected LN metastasis in EGC [5]. However, most of these tumors were predominately of mixed histological type.

To our knowledge, the behavior of histologically mixedtype EGC is incompletely understood, and there is no consensus regarding its clinical management [10, 11]. This study aimed to clarify the clinicopathological features of histologically mixed-type EGC in relation to LN metastasis and to determine indications for endoscopic treatment of mixed-histologic-type EGC.

\section{Patients and methods}

A total of 410 intramucosal gastric cancers in 410 consecutive patients treated surgically between September 2002 and January 2010 in a prefectural cancer center (Shizuoka Cancer Center, Japan) were retrospectively analyzed. Exclusion criteria were: (1) multiple synchronous gastric cancers, (2) local recurrences, (3) unavailable precise pathological records (due to ESD performed at outside hospital, etc.), (4) cancers from remnant stomach, and (5) Barrett cancers. Clinical records and endoscopic and pathological reports of each patient were examined. To assess the histological type, all specimens were reviewed to determine the percentages of differentiated components (well and moderately differentiated tubular adenocarcinoma and papillary adenocarcinoma) and undifferentiated components (poorly differentiated adenocarcinoma, signet ring cell carcinoma, and mucinous adenocarcinoma). Lesions were classified into the following four types according to the proportions of intramucosal differentiated and undifferentiated components at histopathology: pure differentiated (PD) type (composed of differentiated type only), mixed predominantly differentiated (MD) type (major component of differentiated type), mixed predominantly undifferentiated (MU) type (major component of undifferentiated type), and pure undifferentiated (PU) type (undifferentiated type only).

We examined the following parameters: (1) clinicopathological differences between PD- and MD-type tumors, (2) clinicopathological differences between PU type and MU type, and (3) the rate of LN metastasis by tumor size and presence or absence of ulceration. Clinicopathological findings analyzed were: gender, age, predominant histological type, tumor location, macroscopic type, tumor size, presence of lymphatic and venous invasion, presence of ulceration, and LN metastasis. Tumor location was determined based on the Japanese Classification of Gastric Carcinoma [4] as the upper third (U), middle third (M), or lower third (L) of the stomach. Macroscopic type was divided into three groups: elevated type (elevated lesions such as 0-I, and 0-IIa), depressed type (depressed lesions such as 0-IIc), and combined type (such as 0-IIa + IIc and 0-IIc + III). Predominant histological type, tumor size, lymphatic/venous invasion, presence or absence of ulceration, and LN metastasis were determined histopathologically after surgery.

All lesions were thinly sliced at intervals of 3-5 mm. One section each of all dissected LNs (at least 15 nodes per case) was stained with hematoxylin and eosin. Sections were examined histologically to assess the presence or absence of metastasis. Lymphatic invasion was identified immunohistochemically using the D2-40 antibody (DakoCytomation, Glostrup, Denmark). The local ethics committee approved the use of pathological samples, with patients' informed consent, at Shizuoka Cancer Center.

Data were analyzed using the chi-squared test and Student's $t$ test. $P<0.05$ was considered statistically significant.

\section{Results}

\section{Patient characteristics}

Table 1 reports patients' characteristics. The study group consisted of 240 male and 170 female patients with a median age of 61 years (range 29-87 years). Forty-seven lesions were in the upper third of the stomach, 244 in the middle third, and 119 in the lower third. Histopathologically, tumors were divided into four groups as follows: 130 (31.7\%) PD, 54 (13.2 \%) MD, 42 (10.2\%) MU, and 184 (44.9\%) MU. The overall rate of lymphatic invasion was $3.7 \%$, venous invasion was $0 \%$, and $\mathrm{LN}$ metastasis was $7.1 \%$, respectively.

\section{Clinicopathological differences between PD type} and MD type

Table 2 shows relationships between clinicopathological findings and histological type (PD and MD). Moderately differentiated type was observed significantly more often as the main component in MD than in PD lesions (90.7 vs. $46.1 \%, P<0.0001)$. Rates of lymphatic invasion (9.3 vs. $3.1 \%$ ) and LN metastasis (11.1 vs. $3.1 \%$ ) were higher in the MD type than in the PD type, but these differences were not statistically significant. Table 3 shows the 
Table 1 Patients' characteristics

\begin{tabular}{|c|c|}
\hline All & $410(\%)$ \\
\hline \multicolumn{2}{|l|}{ Age (years) } \\
\hline Median (range) & $61(29-87)$ \\
\hline \multicolumn{2}{|l|}{ Gender } \\
\hline Male & $240(58.5)$ \\
\hline Female & 170 \\
\hline \multicolumn{2}{|l|}{ Location } \\
\hline Upper third & $47(11.5)$ \\
\hline Middle third & $244(59.5)$ \\
\hline Lower third & $119(29.0)$ \\
\hline \multicolumn{2}{|l|}{ Macroscopic type } \\
\hline Elevated (I, IIa) & $17(4.1)$ \\
\hline Depressed (IIc) & $346(84.4)$ \\
\hline Complex (IIa + IIc) & $119(11.5)$ \\
\hline \multicolumn{2}{|l|}{ Tumor size (mm) } \\
\hline Median (range) & $33(1-130)$ \\
\hline \multicolumn{2}{|l|}{ Histological type } \\
\hline PD (pure differentiated) & $130(31.7)$ \\
\hline MD (mixed predominantly differentiated) & $54(13.2)$ \\
\hline MU (mixed predominantly undifferentiated) & $42(10.2)$ \\
\hline PU (pure differentiated) & $164(44.9)$ \\
\hline \multicolumn{2}{|l|}{ Lymphatic invasion } \\
\hline Present & $15(3.7)$ \\
\hline Absent & 395 \\
\hline \multicolumn{2}{|l|}{ Vascular invasion } \\
\hline Present & $0(0)$ \\
\hline Absent & 410 \\
\hline \multicolumn{2}{|l|}{ Pathological ulceration } \\
\hline Present & $194(47.3)$ \\
\hline Absent & 216 \\
\hline \multicolumn{2}{|l|}{ LN metastasis } \\
\hline Present & $29(7.1)$ \\
\hline Absent & 381 \\
\hline
\end{tabular}

relationship between lymph node metastases and the pattern of combination of the major differentiated component and the minor undifferentiated component in the MD type. Regarding the minor component, 32 cases were poorly differentiated adenocarcinoma, and 21 cases were signet ring cell carcinoma. There was no significant difference between these minor components and lymph node metastasis.

\section{Clinicopathological differences between PU type and MU type}

Table 4 reports relationships between clinicopathological findings and histological type (PU and MU). Signet ring cell type was observed significantly more often as the main component in the PU versus MU type ( 81.5 vs. $33.3 \%$, $P<0.01)$. LN metastasis was significantly more common in the MU type than in the PU type $(19.0$ vs. $6.0 \%$, $P<0.01$ ). Table 5 shows the relationship between lymph node metastases and the pattern of combination of the major undifferentiated component and the minor differentiated component in the MU type. Most of the minor component was moderately differentiated adenocarcinoma (40/42).

Rate of LN metastasis by tumor size and ulceration

Table 6 shows relationships between LN metastasis and tumor size, presence or absence of ulceration, and histological type. For intramucosal tumors larger than $20 \mathrm{~mm}$ in size without lymphovascular invasion and without ulceration, the rate of $\mathrm{LN}$ metastasis was $0 \%(0 / 17)$ for the $\mathrm{MD}$ type and $24 \%(4 / 17)$ for the MU type. For intramucosal tumors less than $30 \mathrm{~mm}$ with ulceration and without lymphovascular invasion, the rate of LN metastasis was $0 \%$ $(0 / 8)$ for the MD type and $20 \%(2 / 10)$ for the MU type.

\section{Discussion}

New difficulties in determining the appropriate management of mixed histological type gastric cancer arose as a result of improvements in ESD, which allow en-bloc resection of large superficial gastric lesions and precise histopathological evaluation $[10,11]$.

In general, there are two distinct groups of differentiated and undifferentiated mixed-type gastric tumors. The first group has both differentiated- and undifferentiated-type histology in the mucosa. The second group shows differentiated type in the mucosa and undifferentiated type only in the submucosa, which may show features of invasion. We hypothesized that these two groups had different prognoses and should be evaluated and managed independently. For this reason, we investigated only intramucosal gastric cancer in this study.

We histologically classified early gastric cancers into four groups according to the proportion of differentiated and undifferentiated components. We found that mixed tumors with predominantly differentiated type were larger than purely differentiated-type tumors. This is in agreement with studies that reported $86 \%$ of early gastric cancer less than $10 \mathrm{~mm}$ in size were pure differentiated type, and the ratio of mixed type increased with tumor size over $10 \mathrm{~mm}$ [12]. Furthermore, the current study revealed that mixedtype tumors were more often composed of moderately and poorly differentiated histological types than well-differentiated and signet ring cell types. These results suggest that as the tumor grows, moderately differentiated-type lesions might 
Table 2 PD (pure differentiated) type versus MD (mixed predominantly differentiated) type
$S D$ standard deviation

* Chi-squared test, ${ }^{\dagger}$ Student's $t$ test, ${ }^{\S}$ Fisher's exact test

\begin{tabular}{|c|c|c|c|}
\hline$n$ & $\begin{array}{l}\text { PD } \\
130 \quad \%)\end{array}$ & $\begin{array}{l}\text { MD } \\
54(\%)\end{array}$ & $P$ value \\
\hline \multicolumn{4}{|l|}{ Age (years) } \\
\hline Mean \pm SD (range) & $64.2 \pm 9.9(41-83)$ & $61.2 \pm 11.9(29-78)$ & $0.086^{\dagger}$ \\
\hline \multicolumn{4}{|l|}{ Main component } \\
\hline Well differentiated & $69(53.1)$ & $4(7.4)$ & $<0.0001 *$ \\
\hline Moderately differentiated & $60(46.1)$ & $49(90.7)$ & \\
\hline Papillary & $1(0.6)$ & $1(1.9)$ & \\
\hline \multicolumn{4}{|l|}{ Macroscopic type } \\
\hline Elevated (I, IIa) & $14(10.8)$ & $6(1.9)$ & $0.059^{*}$ \\
\hline Depressed (IIc) & $94(72.3)$ & $31(87.0)$ & \\
\hline Complex (IIa + IIc) & $22(16.9)$ & $17(11.1)$ & \\
\hline \multicolumn{4}{|l|}{ Tumor size (mm) } \\
\hline Mean \pm SD (range) & $37.2 \pm 20.2(8-130)$ & $44.4 \pm 30.5(5-127)$ & $0.063^{\dagger}$ \\
\hline \multicolumn{4}{|l|}{ Lymphatic invasion } \\
\hline Present & $4(3.1)$ & $5(9.3)$ & $0.125^{\S}$ \\
\hline Absent & 126 & 49 & \\
\hline \multicolumn{4}{|l|}{ Vascular invasion } \\
\hline Present & $0(0)$ & $0(0)$ & $>0.999^{\S}$ \\
\hline Absent & 130 & 54 & \\
\hline \multicolumn{4}{|l|}{ Pathological ulceration } \\
\hline Present & $63(48.5)$ & $29(53.7)$ & $0.517^{*}$ \\
\hline Absent & 67 & 25 & \\
\hline \multicolumn{4}{|l|}{$\mathrm{LN}$ metastasis } \\
\hline Present & $4(3.1)$ & $6(11.1)$ & $0.066^{*}$ \\
\hline Absent & 126 & 48 & \\
\hline
\end{tabular}

Table 3 The relationship between lymph node metastases and the pattern of combination of the major differentiated component and the minor undifferentiated component in MD type

\begin{tabular}{llll}
\hline MD & \multicolumn{2}{l}{ Minor component } \\
\cline { 2 - 4 } & $\begin{array}{l}\text { Poorly } \\
\text { differentiated }\end{array}$ & $\begin{array}{l}\text { Signet } \\
\text { ring cell }\end{array}$ & Mucinous \\
\hline Major component & & & \\
Well differentiated & $0 / 2$ & $0 / 2$ & 0 \\
Moderately differentiated & $4 / 30$ & $2 / 18$ & 0 \\
Papillary & 0 & $0 / 1$ & 0 \\
\hline
\end{tabular}

progress to poorly differentiated type, and PD type might change into MD type.

Regarding indications for ESD of EGC, MD and PD types are currently managed in the same way as differentiated type tumors according to the Japanese Classification of Gastric Carcinoma [4]. However, our data show that the rate of lymphatic invasion and LN metastasis was higher in the MD type than in the PD type lesions, although the difference was not statistically significant. Hanaoka et al. [10] also reported that the prevalence of LN metastasis was higher with differentiated-type-dominant mixed type (MD type in the current study) than with differentiated type (PD type in the current study). We are apprehensive that MD-type tumors might warrant different treatment protocols from PD-type lesions. The Guidelines for Diagnosis and Treatment of Carcinoma of the Stomach, 3rd edition, were edited by the Japanese Gastric Cancer Society and released in October 2010. In these guidelines there are some modifications concerning the management of mixed histological types. For example, an intramucosal tumor measuring $30 \mathrm{~mm}$ or less, with ulceration, of differentiated-type-dominant mixed type, and without lymphovascular invasion, used to be treated with ESD for curative resection. The new guidelines consider ESD as non-curative resection, because there are no convincing data that these tumor types can be curatively resected. In our study (Table 4), we fortunately found no cases of this tumor type with LN metastasis [MD type, intramucosal, $\leq 30 \mathrm{~mm}$, ulceration $(+)$, lymphovascular invasion $(-)]$, but the number of cases was very small. These findings should be confirmed by additional clinical research.

Hanaoka et al. [10] also reported that the prevalence of lymphatic invasion and LN metastasis was higher with undifferentiated-type-dominant mixed-type tumors (MU in 
Table 4 PU (pure undifferentiated) type versus MU (mixed predominantly undifferentiated) type

$S D$ standard deviation

* Chi-squared test, ${ }^{\dagger}$ Student's $t$ test, ${ }^{\S}$ Fisher's exact test

\begin{tabular}{llll}
\hline$n$ & PU & MU & $P$ value \\
& $184(\%)$ & $42(\%)$ & \\
\hline
\end{tabular}

Age (years)

Mean \pm SD (range)

$59.6 \pm 11.0(30-87)$

$56.7 \pm 11.5(29-81)$

$0.126^{\dagger}$

Main component

Poorly differentiated

34 (18.5)

$14(33.3)$

$0.009^{*}$

Signet ring cell

$150(81.5)$

Mucinous

$0(0)$

27 (64.3)

$1(2.4)$

Macroscopic type

Elevated (I, IIa)

$1(0.5)$

$1(2.4)$

$0.161 *$

Depressed (IIc)

165 (89.7)

40 (95.2)

Complex (IIa + IIc)

$18(9.8)$

$1(2.4)$

Tumor size (mm)

Mean \pm SD (range)

$36.3 \pm 23.3(1-110)$

$36.9 \pm 19.6(8-95)$

$0.883^{\dagger}$

Lymphatic invasion

$\begin{array}{ll}\text { Present } & 4(2.2) \\ \text { Absent } & 180\end{array}$

$2(4.8)$

$0.310^{\S}$

180

40

Present $0(0)$

$0(0)$

$>0.999^{\S}$

Absent

184

42

Pathological ulceration

Present

$81(44.0)$

Absent

103

$21(50.0)$

$0.482 *$

LN metastasis

Present $11(6.0)$

21

8 (19.0)

$0.006^{*}$
Table 5 The relationship between lymph node metastases and the pattern of combination of the major undifferentiated component and the minor differentiated component in MU type

\begin{tabular}{llll}
\hline MU & \multicolumn{2}{l}{ Minor component } \\
\cline { 2 - 4 } & $\begin{array}{l}\text { Well } \\
\text { differentiated }\end{array}$ & $\begin{array}{l}\text { Moderately } \\
\text { differentiated }\end{array}$ & Papillary \\
\hline Major component & & $1 / 13$ & $0 / 1$ \\
Poorly differentiated & $0 / 0$ & $6 / 26$ & 0 \\
Signet ring cell & $1 / 1$ & $0 / 1$ & 0 \\
Mucinous & 0 & & \\
\hline
\end{tabular}

the current study) than with undifferentiated type (PU type in the current study). Our results showed that LN metastases were significantly more common in MU type than in PU type lesions. Although there is a difference between Hanaoka's study, which examined submucosal invasive cancer, and our study examining intramucosal cancer, results should be comparable. That is, MU type tumors might have greater malignant potential than PU tumors.

Limitations of the present study include the retrospective design and inclusion of only surgically resected cases
Table 6 Rate of lymph node metastasis by tumor size and ulcer findings

$M$ intramucosal, ly lymphatic invasion, $v$ vascular invasion, $U L$ ulcer findings

\begin{tabular}{llccc}
\hline $\begin{array}{l}\text { Intramucosal cancer without } \\
\text { lymphovascular invasion } \\
\text { (pM, ly0, v0) }\end{array}$ & $\mathrm{PD}$ & $\mathrm{MD}$ & $\mathrm{MU}$ & $\mathrm{PU}$ \\
\hline $\mathrm{UL}-$ & & & \\
$\quad \leq 20 \mathrm{~mm}$ & $0 / 16(0 \%)$ & $0 / 6(0 \%)$ & $0 / 3(0 \%)$ & $0 / 39(0 \%)$ \\
$>20 \mathrm{~mm}$ & $0 / 49(0 \%)$ & $0 / 17(0 \%)$ & $4 / 17(24 \%)$ & $1 / 62(2 \%)$ \\
$\mathrm{UL}+$ & $0 / 21(0 \%)$ & $0 / 8(0 \%)$ & $2 / 10(20 \%)$ & $2 / 28(7 \%)$ \\
$\leq 30 \mathrm{~mm}$ & $2 / 40(5 \%)$ & $4 / 18(22 \%)$ & $2 / 10(20 \%)$ & $6 / 51(12 \%)$ \\
$>30 \mathrm{~mm}$ & & & &
\end{tabular}


in order to investigate LN metastasis. Furthermore, there is difficulty in determining accurate histopathological diagnoses of mixed type tumors, such as distinguishing between moderately differentiated type and poorly differentiated type lesions. In the present study, all cases were reviewed and diagnosed by at least two pathologists specializing in gastrointestinal pathology.

In conclusion, histologically mixed-type EGC with a predominantly differentiated component might be clinically managed the same way as a differentiated type EGC. However, the rates of lymphovascular invasion and $\mathrm{LN}$ metastasis in MD-type tumors were non-significantly higher than that of PD type lesions. Data suggest that MUtype tumors might have greater malignant potential than PU type tumors. Further investigation is warranted to confirm these findings.

Conflict of interest None.

\section{References}

1. Ono H, Kondo H, Gotoda T, Shirao K, Yamaguchi H, Saito D, et al. Endoscopic mucosal resection for treatment of early gastric cancer. Gut. 2001;48:225-9.

2. Oda I, Gotoda T, Hamanaka H, Eguchi T, Saito Y, Matsuda T, et al. Endoscopic submucosal dissection for early gastric cancer: technical feasibility, operation time and complications from a large consecutive series. Dig Endosc. 2005;17:54-8.

3. Ono H, Hasuike N, Inui T, Takizawa K, Ikehara H, Yamaguchi Y, et al. Usefulness of a novel electrosurgical knife, the insulation- tipped diathermic knife-2, for endoscopic submucosal dissection of early gastric cancer. Gastric Cancer. 2008;11:47-52.

4. Japanese Gastric Cancer Association. Japanese classification of gastric carcinoma: 2nd English edition. Gastric Cancer 1998;1: $10-24$.

5. Gotoda T, Yanagisawa A, Sasako M, Ono H, Nakanishi Y, Shimoda $\mathrm{T}$, et al. Incidence of lymph node metastasis from early gastric cancer: estimation with a large number of cases at two large centers. Gastric Cancer. 2000;3:219-25.

6. Gotoda T, Sasako M, Ono H, Katai H, Sano T, Shimoda T. Evaluation of the necessity for gastrectomy with lymph node dissection for patients with submucosal invasive gastric cancer. Br J Surg. 2001;88:444-9.

7. Yamao T, Shirao K, Ono H, Kondo H, Saito D, Yamaguchi H, et al. Risk factors for lymph node metastasis from intramucosal gastric carcinoma. Cancer. 1996;77:602-6.

8. Kurihara N, Kubota T, Otani Y, Ohgami M, Kumai K, Sugiura H, et al. Lymph node metastasis of early gastric cancer with submucosal invasion. Br J Surg. 1998;85:835-9.

9. Takizawa K, Shimoda T, Nakanishi Y, Taniguchi H, Oda I, Gotoda T. Expanded indication of endoscopic resection from the pathological viewpoint - the ability of sm invasion of early gastric cancer of undifferentiated type. Stomach Intes. 2006;41:9-17.

10. Hanaoka N, Tanabe S, Mikami T, Okayasu I, Saigenji K. Mixedhistologic-type submucosal invasive gastric cancer as a risk factor for lymph node metastasis: feasibility of endoscopic submucosal dissection. Endoscopy. 2009;41:427-32.

11. Takizawa K, Ono H, Hasuike N, Tanaka M, Kusumoto K, Kadooka M, et al. Treatment for early gastric cancer of differentiated and undifferentiated mixed type. Stomach Intes. 2007; 42:1647-58.

12. Saito A, Shimoda T, Nakanishi Y, Ochiai A, Toda G. Histologic heterogeneity and mucin phenotypic expression in early gastric cancer. Pathol Int. 2001;51:165-71. 